\title{
In memoriam Ernst J. Zingg
}

Daniel K. Ackermann, George N. Thalmann, Urs E. Studer

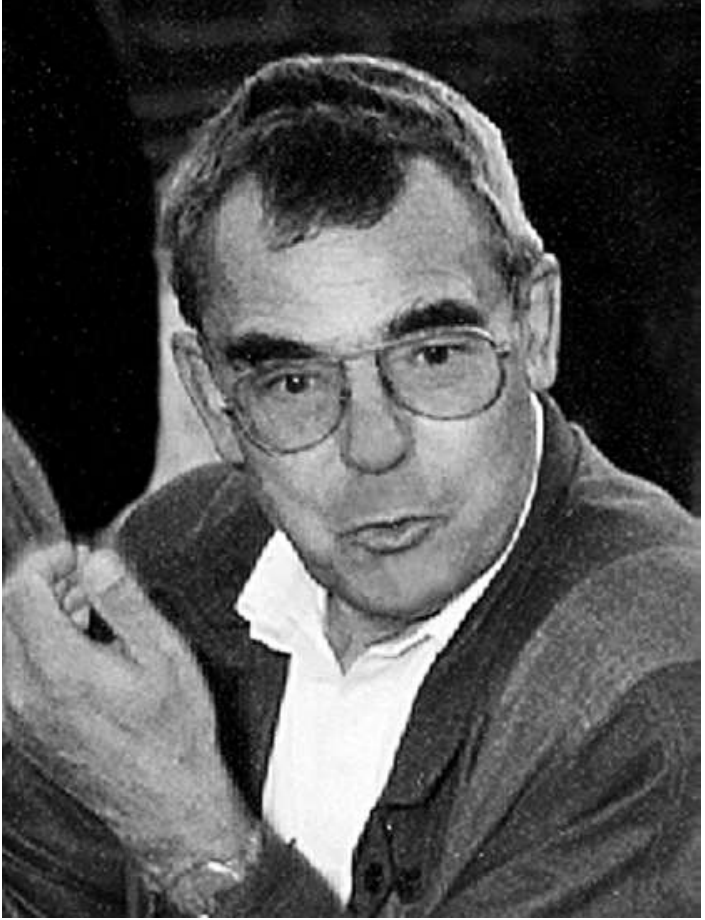

Prof. em. Dr. med. Ernst J. Zingg (1931-2016)
Traurig nehmen wir Abschied von Professor Ernst J. Zingg, einem der Wegbereiter der modernen Urologie in Europa, der am 27. Februar 2016 verstorben ist. Ernst Zingg wurde am 27. Juli 1931 in Zürich geboren. Er war das einzige Kind eines Bauingenieurs und erhielt eine umfassende, konsequente Erziehung, von welcher er zeitlebens profitieren sollte. In Zürich durchlief er alle Schulen und schloss 1956 das Medizinstudium ab. Die erste Assistentenzeit bei Professor H.U. Zollinger, dem damals prominentesten Pathologen der Schweiz, gab ihm nicht nur einen vertieften Einblick in die Anatomie der Weichteile, sondern auch in die Mechanismen der Metastasierung bösartiger Tumoren. In den folgenden 6 Jahren erhielt er die chirurgische Ausbildung wieder bei den zu dieser Zeit bekanntesten Thorax-, Viszeral- und Herzchirurgen der Schweiz, den Professoren A. Brunner und A. Senning. Um ein so anspruchsvolles Ausbildungsprogramm durchzustehen, bedurfte es neben der grossen intellektuellen Fähigkeiten auch der Bereitschaft, bis an die eigene Leistungsgrenze zu gehen. Die Wahl der herausfordernden Spezialarztausbildung war für Ernst J. Zingg charakteristisch, schuf aber auch die Grundlage für seine glänzende Karriere als Kliniker und operativer Urologe.

Nach Erlangung des Spezialarzttitels für Chirurgie wechselte er innerhalb des Zürcher Universitätsspitals zu Professor G. Mayor in die schweizweit erste unabhängige Klinik für Urologie. Mit 34 Jahren wurde er zum Oberarzt ernannt und bereits 4 Jahre später zum Direktor und Chefarzt der Klinik für Urologie am Universitätsspital Bern gewählt, wo er von 1971 bis 1994 tätig war.

\section{Klinischer Lehrer, Forscher und operativer Urologe}

Unter den zahlreichen Publikationen von Ernst Zingg nimmt das Lehrbuch Urologische Operationen, welches er zusammen mit seinem früheren Chef G. Mayor schrieb, eine Sonderstellung ein. Das Buch wurde zum Standardwerk im deutschsprachigen Europa, es wurde ins Spanische und Englische übersetzt und trug wesentlich dazu bei, die Urologie als eigenständiges Fach zu etablieren.

Die Behandlung oberflächlicher und invasiver Blasenkarzinome war einer seiner klinischen und wissenschaftlichen Schwerpunkte. Er propagierte die radikale Zystektomie und präsentierte bereits in den späten $60 e r$ Jahren ein Konzept, welches damals zu einer Senkung der perioperativen Mortalität auf 25\% (!) führte. 1968 erhielt er den Preis der Schweizerischen Gesellschaft für Urologie für die visionäre Habilitationsarbeit über isotope, autologe und homologe Transplantationen der Harnblase. In seiner glanzvollen Karriere

\section{In seiner glanzvollen Karriere erhielt} er verschiedene Preise und Ehrenmitgliedschaften, zu viele, um alle aufzuzählen.

erhielt er verschiedene Preise und Ehrenmitgliedschaften, zu viele, um alle aufzuzählen. Hervorzuheben sind aber die St. Pauls-Medaille der britischen Urologengesellschaft, die Nitze-Medaille der Deutschen und die Ehrenmitgliedschaft der Europäischen Gesellschaft für Urologie. Dank seinem internationalen Re- 
nommee als ausgezeichneter Urologe mit immensem Engagement für die urologische Welt wurde er auch korrespondierendes Mitglied der hoch angesehenen und exklusiven Gesellschaft der American Genito-Urinary Surgeons.

Die profunde Kenntnis über die Geschichte der urologischen Operationen, die mehrjährige Ausbildung in der Viszeral- und Thoraxchirurgie und seine chirurgische Geschicklichkeit waren die Garanten für seinen Erfolg als operativer Urologe, der über die Landesgrenze hinausstrahlte. Hunderte von Urologen aus ganz Europa kamen zu den sogenannten klinischen Wochenenden, die alternierend in Bern und Mainz durchgeführt wurden. Bereits in den 70er Jahren

\section{Aufgrund der exzellenten Resultate wurden Ernst Zingg Nierensteinpatienten von weit her zugewiesen.}

wurde mit Direktübertragungen aus dem Operationssaal in den Hörsaal gearbeitet, wo Moderatoren und Teilnehmer mit den Operateuren jeden chirurgischen Schritt diskutieren konnten. Aufgrund der exzellenten Resultate wurden Ernst Zingg Nierensteinpatienten von weit her zugewiesen zur Entfernung von Nebenschilddrüsenadenomen und komplexen Nierensteinen. Er hatte einen wesentlichen Anteil an der Verbreitung der radikalen Prostatektomie und Zystektomie in Europa und verfeinerte die Technik dieser zwei anspruchsvollen Operationen während seiner ganzen Karriere.

\section{Führungsqualitäten}

Ernst Zingg etablierte in Bern eine straff organisierte Klinik, und nicht von ungefähr begleitete ihn der Ruf, dass der Name Zingg eigentlich mit $3 \mathrm{~K}$ zu schreiben wäre (Kommandieren, Kontrollieren, Korrigieren). Die Abklärung der Patienten, die prä- und postoperative Betreuung waren im klinikinternen "Schwarzbuch" klar definiert und nicht dem Gutdünken des Assistenzoder Oberarztes überlassen. Einmal wöchentlich visitierte er alle stationären Patienten - neben ihm die Oberschwester und sein Stellvertreter, dahinter die Oberärzte und Assistenten. In den Anfangsjahren standen zwar Ultraschall, Computertomographie und Magnetresonanztomographie noch nicht zur Verfügung. Seine grosse Erfahrung und sein klinisches Gespür erlaubten ihm aber auf der Visite, schon im frühen Stadium postoperative Probleme zu erkennen. Ein Arztbericht durfte erst verschickt werden, nachdem der Chef diesen kontrolliert und gegengezeichnet hatte.
Sowohl in der Fakultäts- als auch in der Spitalleitung war er aktiv und setzte sich mit Vehemenz für die Neuorganisation des Universitätsspitals Bern ein. Er befürwortete die Zentralisierung der Pflege bezüglich Ausbildung, Standardisierung und Qualitätsmanagement; für die organisatorischen und administrativen Belange blieben die Pflegenden aber im Verantwortungsbereich der Klinikleitung. Die klare Abgrenzung der Aufgaben und Verantwortlichkeiten dürfte einer der Gründe gewesen sein für die fortwährend gute Zusammenarbeit zwischen Pflegenden und Ärzten am Berner Universitätsspital.

Ernst Zingg realisierte früh, dass zur Verbreitung der modernen Urologie in Europa eine schlagkräftige Europäische Urologengesellschaft (EAU) notwendig war, und bekleidete von 1982 bis 1994 das verantwortungsvolle Amt des EAU-Schatzmeisters. In dieser Funktion war er bei der Ausarbeitung der neuen Statuten massgeblich beteiligt und sorgte dafür, dass Bern zum administrativen Sitz der EAU wurde.

Nach Diagnosestellung einer chronisch progredienten neurologischen Erkrankung entschied sich Ernst Zingg zum vorzeitigen Rücktritt von allen Ämtern. Obwohl

\section{Seine Schüler danken ihm für alles, was sie von ihm lernen durften.}

zu Beginn die Mitarbeiter seine Erkrankung kaum bemerkten, wollte er nicht warten, bis sich bei seiner be ruflichen Tätigkeit krankheitsbedingte Fehler einschleichen würden. So streng und konsequent er mit seinen Mitarbeitern war, so war er es auch mit sich selbst. Nach der Pensionierung blieb er für über 20 Jahre mit der Klinik und den ehemaligen Mitarbeitern freundschaftlich verbunden. Er war ein interessierter Zuhörer, kritisierte nie und gab von sich aus keine Ratschläge zur Klinikführung. Nur wenn er nach seiner Meinung gefragt wurde, äusserte er sich in seiner bekannten, präzisen Art, ohne zu erwarten, dass seine Vorschläge auch umgesetzt würden. Für ihn war es nach seiner Demission selbstverständlich, dass die Zeit als Chef vorbei war.

Leider war Ernst Zingg in den letzten Lebensjahren körperlich zunehmend behindert, was ihn trotz geistiger Frische bei den alltäglichen Aktivitäten stark einschränkte. Seine Frau Monika sorgte mit aufopfernder Hingabe für eine 24-Stunden-Betreuung in seinem geliebten Zuhause. Die Abhängigkeit von anderen war für ihn schwer zu ertragen, er beklagte sich aber nie. Seine Schüler danken ihm für alles, was sie von ihm lernen durften, und werden Ernst J. Zingg als grossartigen Menschen und bedeutenden Urologen in Erinnerung behalten. 